Pacific Journal of Mathematics

BRANCHED IMMERSIONS ONTO COMPACT ORIENTABLE 


\title{
BRANCHED IMMERSIONS ONTO COMPACT ORIENTABLE SURFACES
}

\author{
John D. Elwin AND Donald R. Short
}

\begin{abstract}
In this paper smooth maps $f: M^{n} \rightarrow N^{n}$ with a zerodimensional critical set are considered. The singularities of these maps in the case $n=2$ are known to be points where $f$ is locally topologically equivalent to $z \rightarrow z^{d} \quad(d=$ $2,3, \cdots)$. Originally these singularities were studied in connection with the regularity of Douglas' solution to Plateau's problem.
\end{abstract}

In $\S 1$, an Euler characteristic formula is developed which generalizes both the Riemann-Hurwitz equation from complex analysis and the usual Euler characteristic formula for covering maps. Section 2 is devoted to several technical lemmas while $\S 3$ applies these lemmas to the case where $M$ is the disc (with holes) and $N$ is a compact orientable 2-manifold. It is shown that for the existence of such a map there is a lower limit depending upon the genus of $N$ and on the number of holes of $M$.

The singularities of these maps have been characterized by Church and Timourian [2]. In the case $n=2$ these maps are locally topologically equivalent to $z \rightarrow z^{d}(d=2,3, \cdots)$ and for $n>2$, these maps are covering projections. For $n=2$, the singularities and maps are special cases of branch points and branched immersions. In this case, the Euler characteristic formula represents a generalization of the classical Riemann-Hurwitz equation for light interior transformations on 2-manifolds. When $f^{-1}(\partial N)=\partial M$, the formula produced here reduces to the Riemann-Hurwitz equation. For $n>2$, the maps are covering projections and the Euler characteristic formula reduces to the usual equation.

The mappings considered in this paper are not, in general, interior transformations on the boundary of $M$. These considered here, however, appear to have more applications with regard to questions which have arisen from Plateau's problem. Heinz [6] and Gulliver, Osserman, and Royden [5] have shown that these maps are of much more interest than solely in the context of minimal surfaces.

A very readable account of the Riemann-Hurwitz formula may be found in Whyburn [10]. The authors wish to express their gratitude to the referee for his many helpful suggestions.

1. Let $f: M^{n} \rightarrow N^{n}$ be a continuous map between orientable $n$-manifolds with or without boundary having finite fibres. If $\partial M \neq$ 
$\phi$, assume $f(\partial M)$ is a closed orientable $(n-1)$-submanifold of $N$ without boundary. Let $B$ be any finite subset of $N$. In addition, let us assume that $M, \partial M, N-(f(\partial M) \cup B), f(\partial M)-B$ and $\partial N-$ $(f(\partial M) \cup B)$ have finitely generated homology modules with $\partial N-$ $(f(\partial M) \cup B)$ a closed subset of $N$. For notational convenience we will write the last three spaces as the union of their topological components i.e., $N-(f(\partial M) \cup B)=\bigcup A_{i}, \quad f(\partial M)-B=\bigcup B_{j}$ and $\partial N-(f(\partial M) \cup B)=\cup C_{k}$. We then have the following Euler characteristic relationship:

THEOREM 1. Suppose that under the above assumptions the Leray sheaf is locally constant each component $X\left(X=A_{i}, B_{j}\right.$ or $C_{k}$ ) with stalk given by $\mathscr{H}_{c}^{0}(f ; Z)_{\mid x}=\bigoplus_{\mu(X)} Z$. Further, assume for each component $X$ that $\chi\left(H^{*}\left(X: \mathscr{H}_{c}^{0}(f: Z)\right)=\chi\left(H_{c}^{*}\left(X ; \bigoplus_{\mu^{\prime}(X)} Z\right)\right)\right.$ (here $\chi(\cdots)$ denotes the Euler characteristic). We then have

$$
\begin{aligned}
\chi(M)-\chi(\partial M)= & \sum \mu\left(A_{i}\right) \chi\left(A_{i}\right)-\sum \mu\left(B_{j}\right) \chi\left(B_{j}\right) \\
& -\sum \mu\left(C_{k}\right) \chi\left(C_{k}\right)+(-1)^{n} \sum_{b \in B} \mu(b)
\end{aligned}
$$

where $\mu(b)$ is the cardinality of the fibre over $b$.

Proof. Since $M$ and $N$ are locally compact Hausdorff manifolds, the family of compact supports $c$ is paracompactifying and well adapted [1]. In addition the fibres are $c$-taut in $M$ and thus there is a spectral sequence [1] with

$$
E_{2}^{p, q}=H_{c}^{p}\left(N ; \mathscr{H}_{c}^{q}(f ; Z)\right) \Longrightarrow H_{c}^{p+q}(M ; Z)
$$

and natural isomorphisms

$$
\mathscr{H}_{c}^{*}(f ; Z)_{\mid x} \approx H_{c}^{*}\left(f^{-1}(x) ; Z\right) .
$$

Since covering dimension dominates cohomological dimension [4], the assumption that $f$ is light implies that

$$
E_{2}^{p, q}= \begin{cases}0 & \text { if } q \neq 0 \text { or } p<0 \\ H_{c}^{p}\left(N ; \mathscr{H}_{c}^{0}(f, Z)\right) & \text { otherwise . }\end{cases}
$$

Let us denote $\mathscr{H}_{0}^{0}(f ; Z)$ by $\mathscr{A}$. Clearly this sequence satisfies Serres' theorem [7] which gives the following isomorphism:

$$
H_{c}^{p}(M ; Z) \approx H_{c}^{p}(N ; \mathscr{A}) \text {. }
$$

The sheaf theoretic version of Lefschetz duality [1] implies that

$$
H_{q}(M, \partial M ; Z) \approx H_{c}^{n-q}(M ; Z) .
$$

Combining these isomorphisms and the fact that the homology modules of $M$ and $\partial M$ are finitely generated we obtain 


$$
\chi(M)-\chi(\partial M)=\chi(M, \partial M)=(-1)^{n} \chi\left(H_{c}^{*}(N ; \mathscr{A})\right) .
$$

Thus the proof is reduced to the computation of the sheaf cohomology term.

Since $f(\partial M)$ is a closed $(n-1)$-submanifold in $N$ and $B$ is finite, $f(\partial M) \cup B$ is a closed subset of $N$. Thus there exists the following long exact sequence [1]:

$$
\begin{aligned}
\cdots & \longrightarrow H_{c}^{p}(N-(f(\partial M) \cup B) ; \mathscr{A}) \longrightarrow H_{c_{1}^{p}}^{p}(N ; \mathscr{A}) \longrightarrow \\
& \longrightarrow H_{c}^{p}(f(\partial M) \cup B ; \mathscr{A}) \longrightarrow \\
& \longrightarrow H_{c}^{p+1}(N-(f(\partial M) \cup B) ; \mathscr{A}) \longrightarrow \cdots
\end{aligned}
$$

Let $B_{\partial}=f(\partial M) \cap B$ and $B_{i}=B-B_{\partial}$. Since $B_{i}$ is finite, it is closed in $f(\partial M) \cup B$ and again we have the sequence

$$
\begin{aligned}
\cdots & \longrightarrow H_{c}^{p}(f(\partial M) ; \mathscr{A}) \longrightarrow H_{c}^{p}(f(\partial M) \cup B ; \mathscr{A}) \longrightarrow \\
& \longrightarrow H_{c}^{p}\left(B_{i} ; \mathscr{A}\right) \longrightarrow H_{c}^{p+1}(f(\partial M) ; \mathscr{A}) \longrightarrow \cdots
\end{aligned}
$$

Since $B_{\partial}$ is finite, a third application of the sequence gives

$$
\begin{aligned}
\cdots & \longrightarrow H_{c}^{p}(f(\partial M)-B ; \mathscr{A}) \longrightarrow H_{c}^{p}(f(\partial M) ; \mathscr{A}) \longrightarrow \\
& \longrightarrow H_{c}^{p}\left(B_{\partial} ; \mathscr{A}\right) \longrightarrow H_{c}^{p+1}(f(\partial M)-B ; \mathscr{A}) \longrightarrow \cdots .
\end{aligned}
$$

From the fact that the fibres over $B$ are finite, we have

$$
H_{c}^{0}\left(B_{i} ; \mathscr{A}\right) \approx H^{0}\left(B_{i} ; \mathscr{A}\right) \approx \bigoplus_{b \in B_{i}} H_{0}\left(f^{-1}(b) ; Z\right)
$$

and

$$
H_{c}^{0}\left(B_{\partial} ; \mathscr{A}\right) \approx H^{0}\left(B_{\partial} ; \mathscr{A}\right) \approx \bigoplus_{b \in B_{\partial}} H_{0}\left(f^{-1}(b) ; Z\right)
$$

with all other dimensions trivial.

Using the hypothesis that $f(\partial M)-B$ is an $(n-1)$-manifold without boundary and the Euler characteristic formula for components, Poincaré Duality [1] gives the following equation:

$$
\begin{aligned}
& \chi\left(H_{c}^{*}(f(\partial M)-B ; \mathscr{A})\right)=\sum \chi_{j}\left(H_{c}^{*}\left(B_{j} ; \mathscr{A}\right)\right) \\
& \quad=\sum_{j} \chi\left(H_{c}^{*}\left(B_{j} ; \bigoplus_{\mu\left(B_{j}\right)} Z\right)\right)=(-1)^{n-1} \sum_{j} \mu\left(B_{j}\right) \chi\left(B_{j}\right) .
\end{aligned}
$$

Since the homology modules are finitely generated, we can apply the Euler characteristic formula to each of the above sequences (3), (4), and (5) to obtain

$$
\begin{aligned}
\chi\left(H_{c}^{*}(N ; \mathscr{A})\right)= & \chi\left(H_{c}^{*}(N-(f(\partial M) \cup B) ; \mathscr{A})\right) \\
& +\chi\left(H_{c}^{*}(f(\partial M) \cup B ; \mathscr{A})\right)
\end{aligned}
$$




$$
\begin{aligned}
\chi\left(H_{c}^{*}(f(\partial M) ; \mathscr{A})\right)= & \chi\left(H_{c}^{*}(f(\partial M)-B ; \mathscr{A})\right) \\
& +\chi\left(H_{c}^{*}\left(B_{\partial} ; \mathscr{A}\right)\right) .
\end{aligned}
$$

Combining these equations with (6), (7), and (8) we have

$$
\begin{aligned}
\chi\left(H_{c}^{*}(N ; \mathscr{A})\right)= & \chi\left(H_{c}^{*}(N-(f(\partial M) \cup B) ; \mathscr{A})\right) \\
& +(-1)^{n-1} \sum \mu\left(B_{j}\right) \chi\left(B_{j}\right)+\sum_{b \in B} \mu(b) .
\end{aligned}
$$

Since $\partial N-(f(\partial M) \cup B)$ is closed in $N$, applying the Euler characteristic to the exact sequence of the pair $(N-(f(\partial M) \cup B), \partial N-$ $(f(\partial M) \cup B))$ gives

$$
\begin{aligned}
\chi\left(H_{c}^{*}\right. & (N-(f(\partial M) \cup B) ; \mathscr{A})) \\
& =\sum_{i} \chi\left(H_{c}^{*}\left(A_{i} ; \mathscr{A}\right)\right)+\sum_{k} \chi\left(H_{c}^{*}\left(C_{k} ; \mathscr{A}\right)\right) \\
& =\sum_{i} \chi\left(H_{c}^{*}\left(A_{i} ; \bigoplus_{\mu_{\left(A_{i}\right)}} Z\right)\right)+\sum_{k} \chi\left(H_{c}^{*}\left(C_{k} ; \bigoplus_{\mu\left(C_{k}\right)} Z\right)\right)
\end{aligned}
$$

or by Poincaré Duality

$$
\begin{aligned}
\chi\left(H_{c}^{*}\right. & (N-(f(\partial M) \cup B) ; \mathscr{A})) \\
& =(-1)^{n}\left[\sum_{i}\left(\chi H_{*}\left(A_{i} ; \bigoplus_{\mu\left(A_{i j}^{\prime}\right.} Z\right)\right)-\sum_{k}\left(\chi H_{*}\left(C_{k} ; \bigoplus_{\mu\left(C_{k}\right)} Z\right)\right)\right] \\
& =(-1)^{n}\left[\sum_{i} \mu\left(A_{i}\right) \chi\left(A_{i}\right)-\sum_{k} \mu\left(C_{k}\right) \chi\left(C_{k}\right)\right] .
\end{aligned}
$$

Combining this result with (2) and (9) completes the proof.

2. In this section we will develop several lemmas necessary for the existence theorems of $\S 3$. Recall that a topological submersion is a continuous map $f: M \rightarrow N$ which is locally topologically equivalent to a projection. If $f$ is smooth and its' Jacobian is of maximal rank at each point of $M$, then $f$ is a submersion. The following lemma is a special case of a result proved in [8] for smooth submersions and again in [3] for general submersions.

Lemma A. (Tubular Neighborhoods): If $f: M \rightarrow N$ is a proper surjective submersion between n-dimensional manifolds without boundary then for every $y \in N$ there is a neighborhood $U$ of $y$ and an embedding $\varepsilon: U \times f^{-1}(y) \rightarrow M$ such that

(i) $f \circ \varepsilon$ is projection onto the first factor.

(ii) $\varepsilon(y, x)=x$ for all $x \in f^{-1}(y)$.

(iii) $f^{-1}(U)=\varepsilon\left(U \times f^{-1}(y)\right)$.

Thus each point $y$ of $N$ has a neighborhood $U$ whose inverse image is smoothly equivalent to a product of $U$ with the fibre $f^{-1}(y)$. 
The next lemma follows immediately from the existence of tubular neighborhoods.

Lemma B. (Covering Lemma): Suppose $f: U \rightarrow V$ is a proper surjective submersion between n-manifolds without boundary. Assume that $V$ is connected. Then

(i) the multiplicity of the cover denoted by $\mu(V)$ is constant,

(ii) the Leray sheaf $\mathscr{H}_{c}^{\circ}(f ; Z)$ is locally constant with stalk given by $\mathscr{H}_{c}^{0}(f ; Z)_{\mid x}=\bigoplus_{\mu(V)} Z$.

Lemma $\mathrm{C}$ verifies the Euler characteristic hypothesis of Theorem 1 for certain subsets of compact polyhedra.

LemMA C. Suppose $M$ is a compact polyhedron which is triangulated by the finite simplicial complex $K$. Let $X$ be any subspace of $M$ formed by removing the interior of a number of simplices and a number of vertices. Then for any locally constant sheaf on $X$ with stalk $G$,

$$
\chi\left(H_{c}^{*}(X ; \mathscr{A})\right)=\chi\left(H_{c}^{*}(X: G)\right) .
$$

Proof. Suppose the dimension of $K$ is $m$ and let $L^{i}$ denote the intersection of the $i$-dimensional skeleton of $K$ with $X$. Since $L^{i}$ is a closed subset of $X$ for all $i$, applying the Euler characteristic to the long exact sequence of the pair $\left(L^{i}, L^{i-1}\right)[1]$, we obtain

$$
\chi\left(H_{c}^{*}\left(L^{i} ; \mathscr{B}\right)\right)=\chi\left(H_{c}^{*}\left(L^{i}-L^{i-1} ; \mathscr{B}\right)\right)+\chi\left(H_{c}^{*}\left(L^{i-1} ; \mathscr{B}\right)\right)
$$

for any sheaf $\mathscr{B}$. Hence by induction we have

$$
\chi\left(H_{c}^{*}(X ; \mathscr{B})\right)=\sum_{j=0}^{m} \chi\left(H_{c}^{*}\left(L^{j}-L^{j-1} ; \mathscr{B}\right)\right) \quad \text { where } \quad L^{-1}=\phi .
$$

However, $L^{j}-L^{j-1}$ is the disjoint union of the interiors of simplices, which are simply connected. Since any locally constant sheaf $\mathscr{L}$ is constant on these simply connected sets, we obtain

$$
H_{c}^{*}\left(L^{j}-L^{j-1} ; \mathscr{A}\right) \approx H_{c}^{*}\left(L^{j}-L^{j-1} ; G\right)
$$

and finally

$$
\chi\left(H_{c}^{*}(X ; \mathscr{A})\right)=\chi\left(H_{c}^{*}(X ; G)\right) .
$$

In particular Lemma $C$ applies to subsets of a compact 2-manifold with boundary formed by removing a finite number of points and boundary curves.

Our final lemma gives a description of the behavior of a smooth proper surjection in a neighborhood of the image of a boundary point. 
Lemma D. (Boundary Covering Lemma): Suppose $f: M^{n} \rightarrow N^{n}$ is a proper surjective map between $n$-dimensional nanifolds with boundary having discrete fibres. Assume $f \in C^{1}$ in the interior of $M$ and that $D f$ extends continuously to $\partial M$. Let $\overline{D f}$ denote this extension. Assume $B=f(\{x: \operatorname{det}(\overline{D f}(x))=0\})$ is finite and in addition that $f_{\mid \text {। }}$ is 1-1. Then there exist neighborhoods $U$ of $y \in$ $f(\partial M)-(B \cup \partial N)$ and $V$ of $0 \in R^{n}$ and a local homeomorphism $h: f^{-1} U \rightarrow V$ such that

(i) $h\left(f^{-1} y\right)=0$.

(ii) $h\left(\partial M \cap f^{-1} U\right)=\left\{\left(z_{1}, \cdots, z_{n}\right) \in V: z_{n}=0\right\}$.

(iii) there exists an integer $q>0$ with $\left\{z \in V: z_{n} \geqq 0\right\}$ covered $q+1$ times and $\left\{z \in V: z_{n}<0\right\}$ covered $q$ times by the map $h$.

Proof. Since $f$ is proper the fibres are finite. Suppose $y \notin B$ and let $f^{-1}(y)=\left\{x_{0}, x_{1}, \cdots, x_{q}\right\}$ with $x_{0} \in \partial M$ and $x_{i} \notin \partial M$ for $i>0$. Let $g$ be a chart diffeomorphism mapping the closed upper half plane of $R^{n}$ onto a neighborhood of $x_{0}$. Let $g$ take the bounding hyperplane onto a neighborhood in the boundary of $M$ with $g(0)=x_{0}$. If follows from [9] that there is a $C^{1}$ extension $F$ of $f \circ g$ to all of $R^{n}$ whose derivative agrees with $\overline{D f \circ g}$ on the closed upper half plane. Since $f\left(x_{0}\right)=y \notin B$ we have that $\overline{D F}(0) \neq 0$. Thus by the inverse function theorem there exists a neighborhood $\widetilde{V}$ of the origin in $R^{n}$ and a neighborhood $\widetilde{U}$ of $y \in N$ such that $F_{\mid \widetilde{V}}$ is a $C^{1}$ diffeomorphism onto $\widetilde{U}$ with $\widetilde{U} \cap B=\varphi$. Define $\widetilde{V}_{+}=\tilde{V} \cap R_{+}^{n}$.

Let $x \in \widetilde{U}$ and let $K$ be a compact set satisfying $x \in K \subset \widetilde{U}$. Since $f$ is a proper map $f^{-1}(K)$ is compact in $M$. However, $f^{-1}(K)-$ $g\left(\tilde{V}_{+}\right)$is a closed subset of $f^{-1}(K)$ and hence compact. Thus $f$ restricted to $f^{-1}(\widetilde{U})-g\left(\widetilde{V}_{+}\right)$is proper. From Lemma A there exists a neighborhood $U \subset \widetilde{U}$ such that $f^{-1}(U)-g\left(\tilde{V}_{+}\right)$is homeomorphic to $\left\{x_{1}, \cdots, x_{q}\right\} \times U$. Let $h: f^{-1} U \rightarrow V$ be defined by

$$
h(x)=\left\{\begin{array}{ll}
F^{-1} \circ f(x) & x \in f^{-1} U-g\left(\tilde{V}_{+}\right) \\
g^{-1}(x) & x \in g\left(F^{-1}(U)\right)
\end{array} \text { where } \quad V=F^{-1}(U) \subset \tilde{V} .\right.
$$

3. We wish now to apply equation (2) to branched immersions from the disc or multiply connected disc onto compact orientable 2-manifolds without boundary. Throughout this section we will assume all branched immersions $f: M \rightarrow N$ are surjective and that $f_{\text {।əM }}$ is $1-1$.

Let $D_{m}$ denote the disc with $m$ holes i.e., $\partial D_{m}=V_{i=1}^{m+1} S^{1}$ and let $N$ be a compact orientable 2-manifold without boundary of genus $g$. It follows that 
THEOREM 2. For $g>0$, there does not exist a branched immersion from $D_{2 g-2}$ onto $N$.

The next proposition shows that this result cannot be improved.

Proposition 3. For $g>0$, there does exist a branched immersion from $D_{2 g-1}$ onto $N$.

REMARK 1. The special case of $g=1$ gives $N=T^{2}$ (torus) and it follows as a corollary to Theorem 2 that there does not exist a branched immersion of $D$ onto $T^{2}$.

REMARK 2. It will follow from the proof of Theorem 2 that if there exists an immersion $f: D_{m} \rightarrow N$ with branch points then there does not exist an unbranched immersion $g: D_{m} \rightarrow N$ with the same homotopy type as $f$. Conversely if there exists an immersion $g: D_{m} \rightarrow N$ then there does not exist an immersion with branch points $f: D_{m} \rightarrow N$ of the same homotopy type. The removal of branch points is therefore, a global problem.

The proof of Theorem 2 will require the following two lemmas:

LEMMA E. $\chi(N-f(\hat{\partial} M))=\chi(N)=2-2 g$.

Proof. Since $f$ is $1-1$ on the boundary, $f(\partial M)$ is a disjoint union of 1-spheres $V S_{1}$. Let each $S_{1}$ be expanded to a band $\bar{S}_{1}$ in $N$ with the bands pairwise disjoint. Consider the sequence

$$
\begin{aligned}
\cdots & \longrightarrow H_{n}\left(N-V S_{1}\right) \longrightarrow H_{n}(N) \longrightarrow H_{n}\left(N, N-V S_{1}\right) \longrightarrow \\
& \longrightarrow H_{n-1}\left(N-V S_{1}\right) \longrightarrow \cdots .
\end{aligned}
$$

We have

$$
\chi(N)=\chi\left(N-V S_{1}\right)+\chi\left(N, N-V S_{1}\right) .
$$

Now

$$
H_{n}\left(N, N-V S_{1}\right) \approx H_{n}\left(V \bar{S}_{1}, V \bar{S}_{1}-V S_{1}\right) \text { by excision. }
$$

However, $\chi\left(V \bar{S}_{1}, V \bar{S}_{1}-V S_{1}\right)=\chi\left(V \bar{S}_{1}-V S_{1}\right)-\chi\left(V \bar{S}_{1}\right)$ and the lemma is proved.

Lemma F. Assume the image of a boundary sphere $S^{1} \subset f(\partial M)$ encloses a simply connected region $R$. Further, assume that neither $R$ or its boundary contain any branch point images. Then if $R$ is covered $n$ times there exists a deleted neighborhood of $R$ covered $n+1$ times. 
Proof. From Lemma D it follows that the difference in covering between interior points of $R$ and points in a deleted neighborhood of $R$ is one. Suppose that $R$ is covered one more time than any deleted neighborhood. From Lemmas A and $\mathrm{D}, f_{\mid f^{-1} R}$ is a covering projection. Since $R$ is simply connected this covering projection is trivial. Thus, again by Lemma $\mathrm{D}$, there exists a neighborhood of $R$, say $U$, such that $f^{-1} U=V_{i=1}^{n} U_{i} V \hat{R}$ where each $U_{i}$ is homeomorphic to $U$ via $f$ and $\hat{R}$ is homeomorphic to $R$. Since $\hat{R}$ is a connected component of the closed set $f^{-1} R$ and is also a component of the open set $f^{-1} U$, it is a component of the entire domain which is a contradiction.

Proof of Theorem 2. Assume such an $f: D_{2 g-2} \rightarrow N$ exists.

Case I. $B=\phi$ i.e., $f$ is actually an immersion. In this instance equation (1) reduces to

$$
\chi(M)-\chi(\partial M)=\sum \chi(N-f(\partial M)) \mu(f) .
$$

Let $N-f(\partial M)$ be written as the sum $\sum A_{i}$ of its topological components. We have from Lemma $\mathrm{E}$

$$
\sum \chi\left(A_{i}\right)=\sum \chi(N-f(\partial M))=\chi(N)=2-2 g .
$$

We want to show that

$$
\sum \chi\left(A_{i}\right) \mu\left(A_{i}\right) \leqq \sum \chi\left(A_{i}\right)=2-2 g<3-2 g=\chi(M)-\chi(\partial M) .
$$

Now $\chi\left(A_{i}\right) \leqq 1$ for all $i$. Assume $\chi\left(A_{1}\right)=1$ and $A_{2}$ is the surrounding component of $A_{1}$. From Lemma $\mathrm{F}$ we have $\mu\left(A_{2}\right)>\mu\left(A_{1}\right)$ and $\chi\left(A_{2}\right) \leqq 0$. If $\chi\left(A_{2}\right)=0$ then $A_{2}$ has the homotopy type of a 1 -sphere and there exists a component $A_{3}$ surrounding $A_{2}$ and satisfying $\mu\left(A_{3}\right) \geqq \mu\left(A_{1}\right)$. We may continue in this manner to find a component $A_{j}$ with $\chi\left(A_{j}\right)<0$. If $A_{j}$ serves as the surrounding component for more than one disc then its Euler characteristic is lowered by one for each disc. Hence

$$
\sum \chi\left(A_{i}\right) \mu\left(A_{i}\right) \leqq \sum \chi\left(A_{i}\right)=2-2 g<3-2 g .
$$

Case II. $B \neq \phi$. We want to show that the addition of branch points makes the right hand side of equation (1) more negative.

Since the function $f$ in a neighborhood of a branch point $b$ has the form $z \rightarrow z^{n}$ we have that $\mu(b)<\mu(x)$ for $x$ in a deleted neighborhood of $b$. If $b \in S^{1} \subset f(\partial M)$ then $\chi\left(S^{1}-b\right)=1$ but $\mu(b)<\mu\left(S^{1}-b\right)$. If $b \in A_{i}$ where $A_{i}$ is a component of $N-f(\partial M)$ then $\chi\left(A_{i}-b\right)=$ $\chi\left(A_{i}\right)-1$ and $\mu\left(A_{i}-b\right)>\mu(b)$. 
Let $B_{i}$ be the branch points of $f$ contained in $A_{i}$ and $B_{j}$ the branch points of $f$ in $S_{j}^{1} \in f(\partial M)$. Then by the above equations

$$
\begin{aligned}
\sum_{i} \chi & \left(A_{i}-B_{i}\right) \mu\left(A_{i}-B_{i}\right)-\sum_{j} \chi\left(S_{j}^{1}-B_{j}\right) \mu\left(S_{j}^{1}-B_{j}\right)+\sum_{b \in B} \mu(b) \\
& =\sum_{i} \chi\left(A_{i}\right) \mu\left(A_{i}-B_{i}\right)-\sum_{i}\left|B_{i}\right| \mu\left(A_{i}-B_{i}\right) \\
& =\sum_{j}\left|B_{j}\right| \mu\left(S_{j}^{1}-B_{j}\right)+\sum_{j} \sum_{b_{k} \in B_{j}} \mu\left(b_{k}\right)+\sum_{i} \sum_{b_{k} \in B_{i}} \mu\left(b_{k}\right)
\end{aligned}
$$

where $\left|B_{i}\right|$ denotes the cardinality of $B_{i}$.

$$
\leqq \sum_{i} \chi\left(A_{i}\right) \mu\left(A_{i}-B_{i}\right) \leqq \sum_{i} \chi\left(A_{i}\right)=2-2 g
$$

concluding the proof.

For the proof of Proposition 3 let $M$ be a disc with $2 g-1$ holes and $N$ a surface of genus $g$. Map $M$ onto the complement on the Riemann sphere of a small disc by a linear fractional transformation. The resulting image on the sphere will have $2 g$ holes. Assume onehalf of these are located on the northern hemisphere. With proper alignment we may then identify, with overlap, pairs of holes, one from each hemisphere. The resulting surface is a compact orientable surface of genus $g$ covered once at all points except for a band in the interior of each 2-dimensional hole covered twice. It is easy to see that given a disc with more than $2 g-1$ holes the map described above can be adjusted to map the additional holes onto the bands which are covered twice. There is therefore no upper limit on the number of holes but only a lower limit given by Theorem 2 .

The special case of $g=0$ i.e., $N=S^{2}$ is also of interest. Let $M$ be the disc and $f: M \rightarrow S^{2}$ a branched immersion with $f_{\mid \partial, M} 1-1$ as is assumed throughout this section. $S^{2}-f(\partial M)$ consists of two contractible discs $D_{1}$ and $D_{2}$. Lemma $\mathrm{F}$ shows that $f$ must have branch points.

Let us assume that each branch point of $f$ is covered once and that $D_{1}$ (except at branch points) is covered $n$ times by $f$ while $D_{2}$ is covered $n+1$ times. Denote by $P_{i}, i=1,2$; the collection of branch points in $D_{\imath}$ and let $p_{i}$ be the cardinality of $P_{i}$. Then equation (1) becomes

$$
1=\sum \chi\left(D_{i}-P_{i}\right) \mu\left(D_{i}-P_{i}\right)+\sum_{b \in B} \mu(b)
$$

which reduces to

$$
1=\left(1-p_{1}\right) n+\left(1-p_{2}\right)(n+1)+p_{1}+p_{2} .
$$

The above equation has four possible solutions 
1. $\mu\left(D_{1}-P_{1}\right)=n, \mu\left(D_{2}-P_{2}\right)=n+1 ; p_{1}=0, p_{2}=2$.

2. $\mu\left(D_{1}-P_{1}\right)=2, \mu\left(D_{2}-P_{2}\right)=3 ; \quad p_{1}=2, p_{2}=1$.

3. $\mu\left(D_{1}-P_{1}\right)=3, \mu\left(D_{2}-P_{2}\right)=4 ; \quad p_{1}=3, p_{2}=0$.

4. $\mu\left(D_{1}-P_{1}\right)=2, \mu\left(D_{2}-P_{2}\right)=3 ; \quad p_{1}=4, p_{2}=0$.

The first solution is possible and is Example 5.2 in [5]. Solutions 3 and 4 are ruled out by Lemma $F$. The second possibility is also eliminated by the observation that $D_{2}$ has a single branch point and since $D_{2}$ is covered 3 times, $f$ must have the form $z \rightarrow z^{3}$ on $D_{2}$. It follows that $f_{\mid \supset M}$ cannot be $1-1$. Hence there is, up to winding, only one branched immersion from $D$ to $S^{2}$ with the branch points covered once.

\section{REFERENCES}

1. G. E. Bredon, Sheaf Theory, McGraw-Hill, New York, 1967.

2. P. T. Church and J. G. Timourian, Differentiable maps with 0-dimensional critical set, Pacific J. Math., 41 (1972), 615-630.

3. D. B. Gauld, Tubular neighborhoods for submersions of topological manifolds, University of Auckland report No. 11, Auckland, N. Z., 1972.

4. R. Godement, Topologie Algébrique et Theorie des Faisceaux, Hermann, Paris, 1964.

5. R. Gulliver, R. Osserman, and H. Royden, A theory of branched immersions of surfaces, Amer. J. Math., 95 (1973), 750-812.

6. E. Heinz, Ein Regularitätssatz für Flächen beschränkter mitterer Krümmung, Nachr. Akad. Wiss. Göttingen Math.-Phys. Kl. 2 (1969), 107-118.

7. J. P. Serre, Homologie singulière des espaces fibrés, Applications, Ann. of Math., 2 (1951), 425-505.

8. D. R. Short and J. W. Smith, A Vietoris-Begle theorem for submersions, Indiana Univ. Math. J., 10 (1970), 327-336.

9. H. Whitney, Functions differentiable on the boundaries of regions, Ann. of Math., 34 (1934), 482-485.

10. G. T. Whyburn, Analytic Topology, American Mathematical Society Colloquium Publication vol. 28, New York, 1942.

Received April 20, 1973 and in revised form September 13, 1973.

California State University, San Diego 


\section{PACIFIC JOURNAL OF MATHEMATICS}

\section{EDITORS}

RICHARD ARENS (Managing Editor)

University of California

Los Angeles, California 90024

R. A. Beaumont

University of Washington

Seattle, Washington 98105
J. DugundJI

Department of Mathematics University of Southern California Los Angeles, California 90007

D. Gilbarg AND J. Milgram Stanford University

Stanford, California 94305

\section{ASSOCIATE EDITORS}
E. F. BECKENBACH
B. H. NeUmanN
F. WOLF
K. YOSHIDA

\section{SUPPORTING INSTITUTIONS}

UNIVERSITY OF BRITISH COLUMBIA CALIFORNIA INSTITUTE OF TECHNOLOGY

UNIVERSITY OF CALIFORNIA

MONTANA STATE UNIVERSITY

UNIVERSITY OF NEVADA

NEW MEXICO STATE UNIVERSITY

OREGON STATE UNIVERSITY

UNIVERSITY OF OREGON

OSAKA UNIVERSITY
UNIVERSITY OF SOUTHERN CALIFOF

STANFORD UNIVERSITY

UNIVERSITY OF TOKYO

UNIVERSITY OF UTAH

WASHINGTON STATE UNIVERSITY UNIVERSITY OF WASHINGTON

AMERICAN MATHEMATICAL SOCIET NAVAL WEAPONS CENTER 


\section{Pacific Journal of Mathematics}

\section{Vol. 54, No. 1 \\ May, 1974}

Ralph K Amayo, Engel Lie rings with chain conditions ..................

Bernd Anger and Jörn Lembcke, Hahn-Banach type theorems for hypolinear

functionals on preordered topological vector spaces ..................

Gregory Frank Bachelis and Samuel Ebenstein, On $\Lambda(p)$ sets ................

Harvey Isaac Blau, Indecomposable modules for direct products of finite

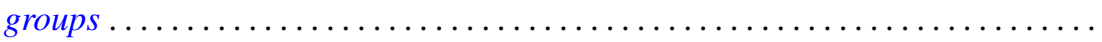

Larry Eugene Bobisud and James Calvert, Singular perturbation of a

time-dependent Cauchy problem in a Hilbert space ................

Walter D. Burgess and Robert Raphael, Abian's order relation and orthogonal

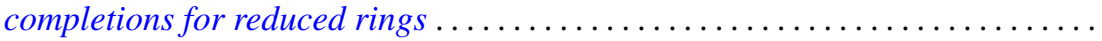

James Diederich, Representation of superharmonic functions mean continuous at

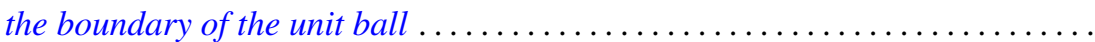

Aad Dijksma and Hendrik S. V. de Snoo, Self-adjoint extensions of symmetric

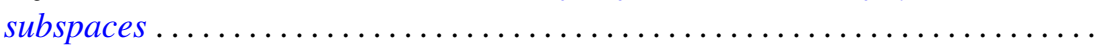

Gustave Adam Efroymson, A Nullstellensatz for Nash rings . . . . . . . . . . . . .

John D. Elwin and Donald R. Short, Branched immersions onto compact orientable surfaces . . . . . . . . . . . . . . . . . . . . . . . . .

John Douglas Faires, Comparison of the states of closed linear

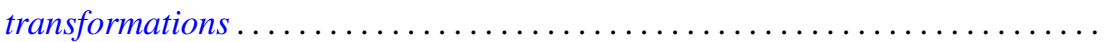

Joe Wayne Fisher and Robert L. Snider, On the von Neumann regularity of rings with regular prime factor rings .

Franklin Takashi Iha, A unified approach to boundary value problems on compact intervals

Palaniappan L. Kannappan and Che Tat $\mathrm{Ng}$, On functional equations connected with directed divergence, inaccuracy and generalized directed divergence

Samir A. Khabbaz and Elias Hanna Toubassi, The module structure of Ext $(F, T)$ over the endomorphism ring of $T$...

Garo K. Kiremidjian, On deformations of complex compact manifolds with boundary.

Dimitri Koutroufiotis, Mappings by parallel normals preserving principal

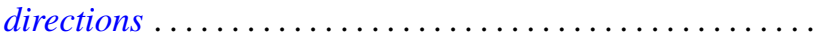

W. K. Nicholson, Semiperfect rings with abelian adjoint group

Norman R. Reilly, Extension of congruences and homomorphisms to translational hulls

Sadahiro Saeki, Symmetric maximal ideals in $M(G)$

Brian Kirkwood Schmidt, On the homotopy invariance of certain functors ...

H. J. Shyr and T. M. Viswanathan, On the radicals of lattice-ordered rings ...

Indranand Sinha, Certain representations of infinite group algebras ...

David Smallen, The group of self-equivalences of certain complexes ...

Kalathoor Varadarajan, On a certain problem of realization in homotopy

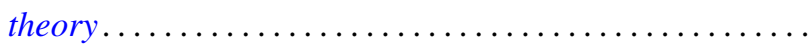

